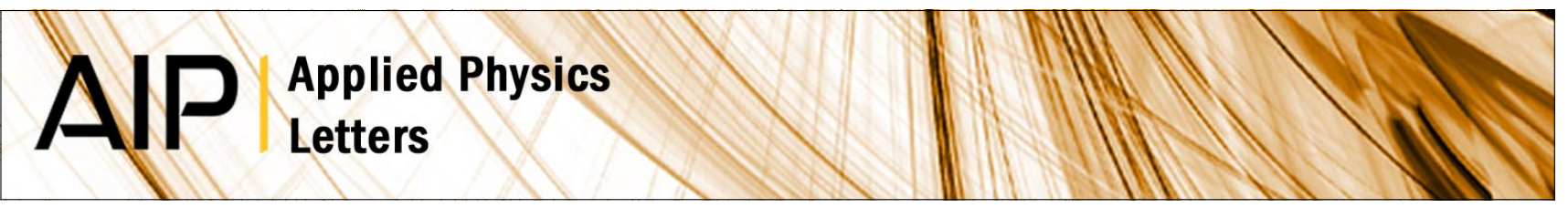

\title{
A level set simulation for ordering of quantum dots via cleaved-edge overgrowth
}

\author{
X. B. Niu, E. Uccelli, A. Fontcuberta i Morral, and C. Ratsch
}

Citation: Appl. Phys. Lett. 95, 023119 (2009); doi: 10.1063/1.3182730

View online: http://dx.doi.org/10.1063/1.3182730

View Table of Contents: http://apl.aip.org/resource/1/APPLAB/v95/i2

Published by the American Institute of Physics.

\section{Related Articles}

Quantitative strain mapping of InAs/InP quantum dots with $1 \mathrm{~nm}$ spatial resolution using dark field electron holography

Appl. Phys. Lett. 99, 261911 (2011)

Preparation of uncapped CdSe1xSx semiconducting nanocrystals by mechanical alloying

J. Appl. Phys. 110, 124306 (2011)

Comparing retention and recombination of electrically injected carriers in Si quantum dots embedded in Si-rich SiNx films

Appl. Phys. Lett. 99, 243501 (2011)

Dilute-nitride GalnAsN/GaAs site-controlled pyramidal quantum dots

Appl. Phys. Lett. 99, 181113 (2011)

Dynamics of interatomic Coulombic decay in quantum dots

J. Chem. Phys. 135, 144112 (2011)

\section{Additional information on Appl. Phys. Lett.}

Journal Homepage: http://apl.aip.org/

Journal Information: http://apl.aip.org/about/about_the_journal

Top downloads: http://apl.aip.org/features/most_downloaded

Information for Authors: http://apl.aip.org/authors

\section{ADVERTISEMENT}

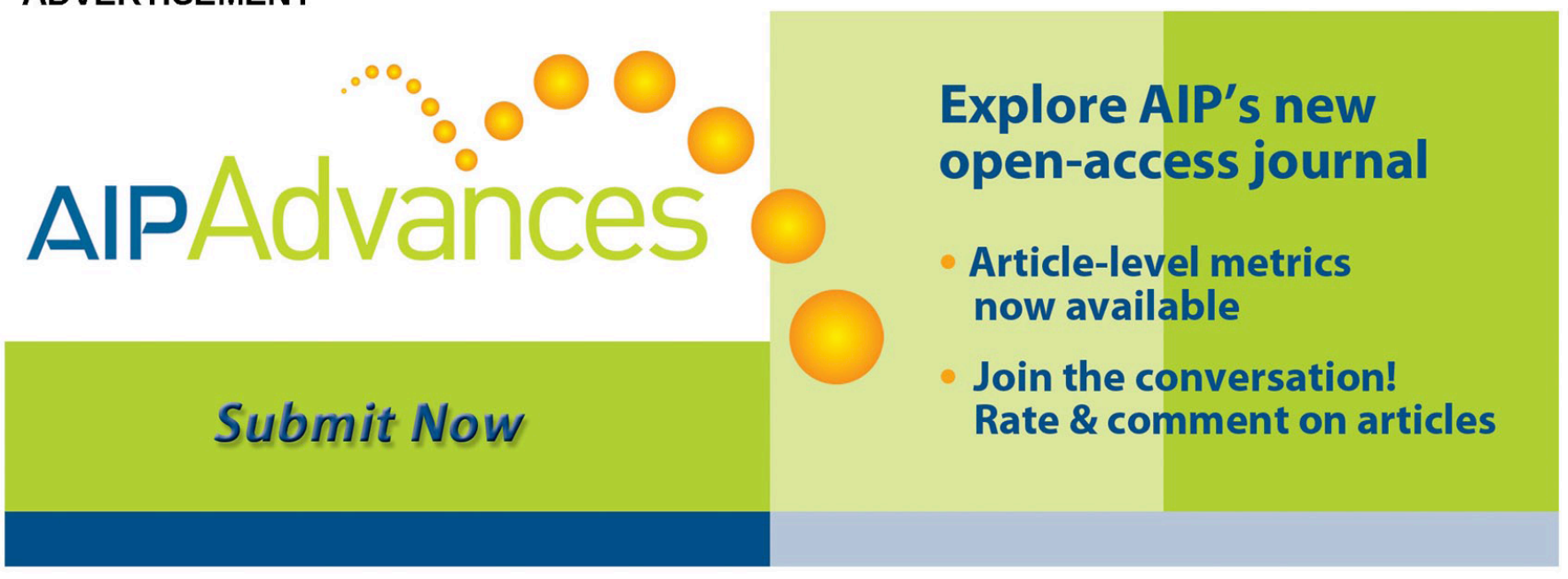




\title{
A level set simulation for ordering of quantum dots via cleaved-edge overgrowth
}

\author{
X. B. Niu, ${ }^{1,2}$ E. Uccelli, ${ }^{3,4}$ A. Fontcuberta i Morral, ${ }^{3}$ and C. Ratsch ${ }^{5, a)}$ \\ ${ }^{1}$ Department of Material Sciences and Engineering, UCLA, Los Angeles, California 90095, USA \\ ${ }^{2}$ Department of Materials Sciences and Engineering, University of Utah, Salt Lake City, Utah 84112, USA \\ ${ }^{3}$ Laboratoire des Matériaux Semiconducteurs, Institut des Matériaux, Ecole Polytechnique Fédérale \\ de Lausanne, CH-1015 Lausanne, Switzerland \\ ${ }^{4}$ Walter Schottky Institute, Technische Universität München, D-85748 Garching, Germany \\ ${ }^{5}$ Department of Mathematics, UCLA, Los Angeles, California 90095, USA and Institute for Pure and \\ Applied Mathematics, UCLA, Los Angeles, California 90095, USA
}

(Received 27 April 2009; accepted 25 June 2009; published online 17 July 2009)

Cleaved-edge overgrowth (CEO) is a promising technique to obtain ordered arrays of quantum dots, where the size and position of the dots can be controlled very well. We present level set simulations for CEO. Our simulations illustrate how the quality of the CEO technique depends on the potential energy surface (PES) for adatom diffusion, and thus suggest how variations of the PES can potentially improve the uniformity of quantum dot arrays. (C) 2009 American Institute of Physics. [DOI: $10.1063 / 1.3182730]$

Semiconductor quantum dots (QDs) have attracted a lot of attention in the last couple of decades and hold great promise for many technological applications such as next generation optoelectronic devices. ${ }^{1}$ For optimal performance of arrays of QDs it is ideal if the size distribution of the dots is rather uniform. Moreover, the exact properties of QDs can be modified by changing its size. It is therefore of paramount importance in the fabrication process of such QDs and arrays of QDs to be able to control their sizes and locations.

Recently, some of us ${ }^{2}$ have introduced a way to control the positioning of QDs during growth based on the cleavededge overgrowth (CEO) technique. We briefly review the method here, but refer to Ref. 3 for more details. A structure with alternating thin films of GaAs and AlAs is grown epitaxially on a (001) oriented GaAs substrate. The films can be as thin as just a few nanometers. After growth of a number of these structures, we cleave the crystal in situ along the [110] direction. As a result, we now have a surface that is flat and patterned with alternating stripes of $\operatorname{GaAs}(110)$ and AlAs(110). For the results that are shown in Figs. 1(a) and 1(b), the GaAs stripes are all $50 \mathrm{~nm}$ wide, while the width of the AlAs stripes varies between 32 and $40 \mathrm{~nm}$; in Fig. 1(c) a single AlAs stripe is shown that is $100 \mathrm{~nm}$ thick. If the cleavage is done carefully, the surface is essentially free of defects, and the interfaces between the GaAs and AlAs stripes are well defined.

We then grow experimentally $0.75-1.0$ (monolayer) ML of InAs on top of this substrate. The resulting morphology is rather striking; at the right growth conditions, all the InAs accumulate on top of the AlAs. Rows of InAs dots form, which are almost perfectly straight. Up to a certain width of the AlAs stripes [Figs. 1(a) and 1(b)], the lateral size of the dots correspond to the width of the AlAs stripe. For AlAs stripes that are wider [Fig. 1(c)], two rows of InAs dots form. The natural question that arises is: what is the driving force for the preferred nucleation on top of the AlAs stripes?

\footnotetext{
${ }^{a)}$ Electronic mail: cratsch@math.ucla.edu.
}

To answer this question, we have performed simulations of growth on a substrate with a spatially varying potential energy surface (PES) that is based on the level set technique. ${ }^{4-6}$ A typical PES with a varying adsorption energy $E_{\text {ad }}$ and transition energy $E_{\text {trans }}$ is sketched in Fig. 2. Within the method, island boundaries are described by a level set function, and growth of islands is described via a velocity of the island boundaries that is proportional to the gradient of the adatom concentration. The adatom concentration $\rho$ is obtained from solving the diffusion equation,

$$
\frac{\partial \rho}{\partial t}=F+\nabla \times(\mathbf{D} \nabla \rho)-2 \frac{d N}{d t}+\nabla \times\left(\frac{\rho}{k_{B} T} \mathbf{D}\left(\nabla E_{\mathrm{ad}}\right)\right) .
$$

In Eq. (1), $\mathbf{D}$ is a diffusion tensor, $F$ is the deposition flux, $d N / d t$ is the nucleation rate, and the last term is the thermodynamic drift, where $k_{B}$ is the Boltzmann constant and $T$ is the temperature. We solve Eq. (1) with a boundary condition $\rho(\mathbf{x})=\rho_{\mathrm{eq}}\left[D_{\mathrm{det}}(\mathbf{x}), \mathbf{x}\right]$, where $D_{\mathrm{det}}(\mathbf{x})$ is a (spatially varying) detachment rate. ${ }^{7}$

We choose a diffusion tensor $\mathbf{D}$ that has only diagonal entries $D_{i}(\mathbf{x})$ and $D_{j}(\mathbf{x})$ that correspond to the spatially vary-

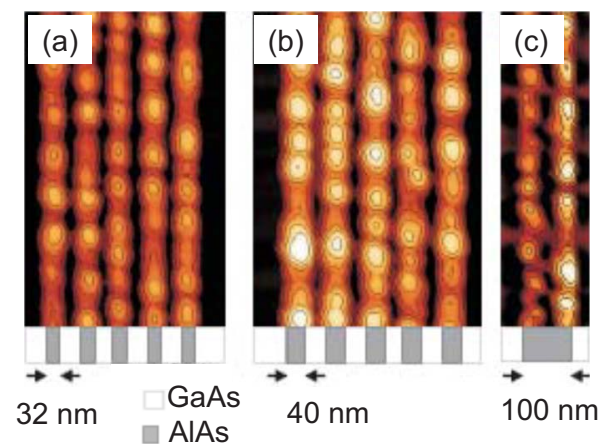

FIG. 1. (Color online) Atomic force micrographs (AFMs) of cleaved (110) facets overgrown by nominally 1.0ML [(a) and (b)] and 0.75ML (c) of InAs, whose height is indicated by contour plots. The surfaces are patterned by AlAs and GaAs stripes of different sequences, as sketched below each AFM. The deposition flux is between 0.018 and $0.028 \mathrm{ML} \mathrm{s}^{-1}$, and the growth temperature is between 710 and $740 \mathrm{~K}$. 


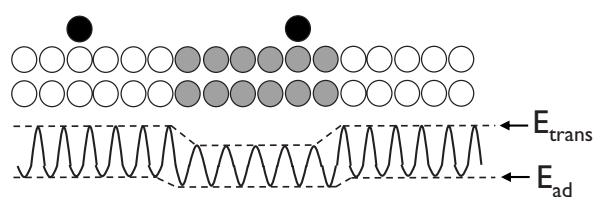

FIG. 2. Sketch of a PES. The black atoms indicate the In adatom, on top of a GaAs(110) surface (white atoms), and an AlAs(110) surface (gray atoms). The dashed line is the envelope of the transition energies $E_{\text {trans }}$ and adsorption energies $E_{\mathrm{ad}}$ of the PES. The PES shown in Fig. 3 refers to this envelope.

ing diffusion along the two directions, $i$ and $j$. These terms are of the form $D=D_{0} \exp \left(-\Delta E / k_{B} T\right)$, where $D_{0}$ is a prefactor (chosen to be $10^{13} \mathrm{~s}^{-1}$ ) and $\Delta E$ is the energy barrier for surface diffusion given by $\Delta E=E_{\text {trans }}-E_{\text {ad }}$. The nucleation rate is given by ${ }^{5}$

$$
d N / d t=\sigma_{1}\left\langle\left\{\left[D_{i}(\mathbf{x})+D_{j}(\mathbf{x})\right] / 2\right\} \rho^{2}(\mathbf{x})\right\rangle,
$$

where $\sigma_{1}$ is the so-called capture number ${ }^{8}$ and the average $\langle\cdot\rangle$ is taken over all lattice sites.

The basic idea and assumption of our model for CEO is that the atomic structure of the $\mathrm{GaAs}(110)$ and $\mathrm{AlAs}(110)$ stripes are the same (i.e., we ignore potentially different reconstructions), and that then the main effect is that $E_{\mathrm{ad}}$ and $E_{\text {trans }}$ for an In adatom on the two different substrates are different. This assumption is supported by results for several different surfaces. For example, density-functional theory (DFT) by calculations by Penev et al. ${ }^{9}$ indicate that the diffusion barrier for In on the $\operatorname{In}_{2 / 3} \mathrm{Ga}_{1 / 3} \mathrm{As}(100)$ surface is approximately $0.3 \mathrm{eV}$, while it is $0.65 \mathrm{eV}$ on the GaAs(001)-c(4×4) surface. Experimental results of Ballet et al. ${ }^{10}$ suggest that diffusion of In adatoms on the $\mathrm{GaAs}(001)$ surface is faster than on the AlAs(001) surface. Formation of $\operatorname{In}_{x} \mathrm{Ga}_{1-x} \mathrm{As}$ islands on $\mathrm{GaAs}(110)$ and the mobility of In on $\operatorname{GaAs}(110)$ have been studied in Refs. 11 and 12 .

We are not aware of any comparison of the mobility of In adatoms on $\operatorname{AlAs}(110)$ and $\operatorname{GaAs}(110)$. However it is evident from Eq. (2) that nucleation will be enhanced if either $D$ or $\rho$ is larger in a particular region. We therefore test either of these two scenarios, and a combination of both cases. The results are shown in Fig. 3. They were obtained for systems of size $400 \times 400$ lattice sites. For all results shown, the deposition flux $F$ is $0.1 \mathrm{ML} \mathrm{s}^{-1}, T=700 \mathrm{~K}$, and $\Delta E=E_{\text {trans }}$ $-E_{\mathrm{ad}}=0.972$ in the GaAs regions such that $D / F=10^{7}$. The values for $\Delta E$ in the AlAs regions are given below. $D_{\text {det }}$ is chosen to be constant with the same value in all regions. We have checked that spatial variations of $D_{\text {det }}$ (and therefore spatial variations in island stabilities) have essentially no effect and cannot explain the preferred island formation in the AlAs regions. The AlAs stripes are between 10 and 30 lattice sites wide, which correspond to a width between 5 and $15 \mathrm{~nm}$. The widths of the GaAs and AlAs regions are slightly smaller than in the experiments, and correspondingly we have chosen values for $D / F$ that are slightly smaller. Nevertheless, we believe that the physical trends are properly captured. We did not choose larger values for $D / F$ (and correspondingly a larger system) for numerical reasons.

In Fig. 3 we show the surface morphologies after the deposition of 0.2 ML (top panels), the envelope of the assumed PES (middle panels), and the nucleation rate calculated according to Eq. (2) just before and after nucleation of
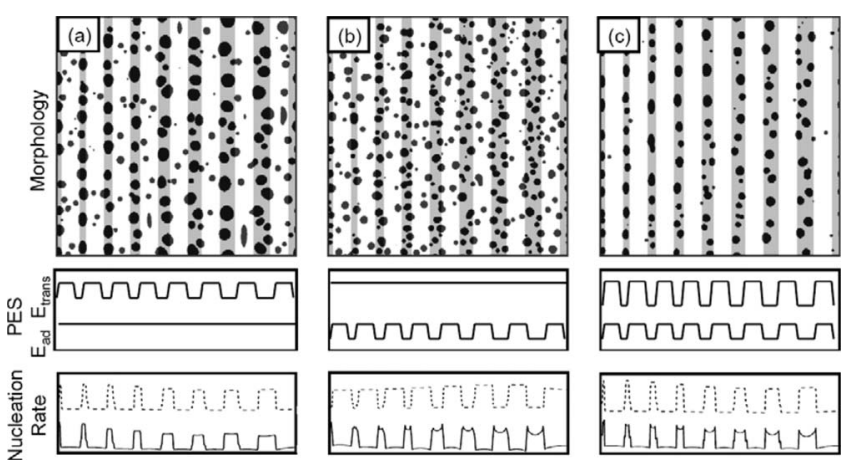

FIG. 3. Simulation results for lower transition energy (i.e., faster diffusion) in $\operatorname{AlAs}(110)$ stripes (a), lower adsorption energy in $\operatorname{AlAs}(110)$ stripes (b), and simultaneously faster diffusion and lower adsorption energy (c). Shown are island morphologies at a coverage of $20 \%$ (top panels), a schematic of the PES (middle panels), and the nucleation rate as a function of the $x$-position just before (dashed line) and after (solid line) nucleation of the first island (bottom panels). Note that the nucleation rate before the first nucleation event (dashed line) is shifted vertically.

the first island (bottom panels). In (a) we show results that correspond to a faster adatom diffusion in the AlAs regions. More precise, we lower $E_{\text {trans }}$ by $0.1 \mathrm{eV}$ in the AlAs region, which leads to an increase in $D_{i}$ and $D_{j}$ by a factor of $\sim 5$. For simplicity, we assume isotropic diffusion along the $i$ and $j$ directions. In (b), we show results that correspond to a higher adatom concentration in the AlAs regions. They were obtained by lowering $E_{\text {ad }}$ in the AlAs regions by $0.1 \mathrm{eV}$. Note that in (b), In adatoms are actually slower in the AlAs regions, by a factor of $\sim 5$ (because we keep $E_{\text {trans }}$ constant). The value of $0.1 \mathrm{eV}$ was chosen because it is larger than $k T$ and because results of Refs. 9 and 12 for diffusion of different species on $\operatorname{GaAs}(110)$ and for diffusion of In on $\mathrm{In}_{2 / 3} \mathrm{Ga}_{1 / 3} \mathrm{As}(100)$ suggest that variations of this order are reasonable for diffusion of In on different (110) surfaces. We observe similar results for slightly different energy variations, but note that we found that systems with energy variations that are significantly less than $k T$ (by an order of magnitude or more) do not exhibit the ordering we present here.

It is evident that in both cases (a) and (b) we get preferred nucleation along the AlAs stripes. The islands in (a) are significantly bigger because diffusion is faster, and thus a typical diffusion length (or island separation) is larger. The discrepancy in the island sizes can of course be avoided if we assumed an overall slower or faster diffusion in (a) or (b), respectively (which can be obtained in the experiment by changing $T$ ), or by changing $F$ to change the ratio $D / F$.

In Figs. 3(a) and 3(b) we still see non-negligible nucleation in the GaAs regions. In (a), there is preferred nucleation in the AlAs regions because of faster diffusion. This can also be seen by focusing on the nucleation rate (bottom panels of Fig. 3). The nucleation rates are significantly higher in the AlAs regions. However after nucleation of the first islands (in the AlAs regions), adatoms are getting depleted there, so that the relative probability for islands to nucleate in the GaAs regions increases (because even though adatoms diffuse slower here, there are more of them).

In (b), the higher In adatom concentration in the AlAs regions is a driving force for preferred nucleation here. However it is competing with a driving force for preferred nucleation in the GaAs regions because of the faster diffusion there. In fact, just before nucleation of the first island, the 
nucleation rate is higher in the GaAs regions (cf. dashed line, bottom panel), and only a little later is the nucleation rate higher in the AlAs regions (solid line, bottom panel). We note that this reversal of the nucleation rate is not present in (a) and (c). These observations suggest that one should expect best alignment of islands in the AlAs regions if there is simultaneously a higher adatom concentration due to a lower $E_{\text {ad }}$, and faster mobility of the In adatoms due to a lower $E_{\text {trans }}$.

We therefore present results in Fig. 3(c) where diffusion of In is faster on the AlAs(110) regions, and at the same time the chemical potential of In adatoms is lower in this region. This is the result of a lowered $E_{\mathrm{ad}}$ (by $0.1 \mathrm{eV}$ ) and a lowered $E_{\text {trans }}$ (by $0.2 \mathrm{eV}$ ). All the nucleation occurs in the AlAs regions. Moreover, when the AlAs stripes are narrow (left side), all the islands are nicely aligned, and there are no islands side-by-side. On the other hand, when the AlAs stripes are wide, we see that some islands have nucleated on the left edge of the AlAs stripe, others on the right edge, and that there are in fact many cases where two islands are nucleated side-by-side. We believe that an explanation for this effect might be that nucleation of two islands side by side occurs when the widths of the stripes are comparable to the diffusion length, and that then the islands tend to nucleate as far away as possible from previously nucleated islands, which is at the edge of the stripes. In addition, the islands grow away from each other (toward the edges of the stripes) because there are more adatoms available on these sides to attach to the islands. For stripes with intermediate widths we see a transition between the two scenarios described. Our results therefore agree very well with the experimental observations in Fig. 1.

The results presented in Fig. 3(c) agree best with the experimental results in Fig. 1. We note, however, that we cannot be certain that the PES for the GaAs(110)/AlAs(110) system exhibits indeed the behavior assumed in Fig. 3(c). While we believe that such a PES is quite plausible, it is also possible that for this particular system preferred nucleation in the AlAs regions is a result of faster diffusion, or a lower chemical potential, as illustrated in Figs. 3(a) and 3(b), and that a potential competing effect as discussed above is simply not relevant. For example, Refs. 10 and 13 assumed that the In adatom diffusion is faster in the GaAs regions; this is possible, as long as a lower chemical potential (or lower $E_{\mathrm{ad}}$ ) in the AlAs regions dominates. However the simulations presented here have given us some deeper insight into the interplay of the adatom diffusivity and nucleation rate, and its effect on the growth mechanism for CEO based arrays of QDs. In particular, our results suggest that systems with a PES that resemble the one assumed in Fig. 3(c) are best suited for CEO. Therefore, our results open the door for exploring other material systems to use this method for getting aligned QDs.

This research was supported in part by the MARCO Center on Functional Engineered NanoArchitectonics (FENA), by the NSF through Grant Nos. DMS-0402276 and DMS-0439872, and in part by the DFG (in the SFB $631 \mathrm{TP}$ B1 and the Nanosystems Initiative Munich-NIM), by the BmBF through Grant No. NanoQUIT-01BM469, and by the Marie Curie Excellence Grant "SENFED."

${ }^{1}$ J. Stangl, V. Holy, and G. Bauer, Rev. Mod. Phys. 76, 725 (2004).

${ }^{2}$ E. Uccelli, M. Bichler, S. Nürnberger, G. Abstreiter, and A. Fontcuberta i Morral, Nanotechnology 19, 045303 (2008).

${ }^{3}$ E. Uccelli, J. Bauer, M. Bichler, D. Schuh, J. J. Finley, G. Abstreiter, and A. Fontcuberta i Morral, Self-Assembled Quantum Dots (Springer, Berlin, 2008), pp. 25-41.

${ }^{4}$ M. Petersen, C. Ratsch, R. E. Caflisch, and A. Zangwill, Phys. Rev. E 64, 061602 (2001).

${ }^{5}$ C. Ratsch, M. F. Gyure, R. E. Caflisch, F. Gibou, M. Petersen, M. Kang, J. Garcia, and D. D. Vvedensky, Phys. Rev. B 65, 195403 (2002).

${ }^{6}$ X. Niu, R. Vardavas, R. E. Caflisch, and C. Ratsch, Phys. Rev. B 74, 193403 (2006).

${ }^{7}$ R. E. Caflisch, W. E, M. F. Gyure, B. Merriman, and C. Ratsch, Phys. Rev. E 59, 6879 (1999).

${ }^{8}$ J. Venables, Philos. Mag. 27, 697 (1973); G. S. Bales and D. C. Chrzan, Phys. Rev. B 50, 6057 (1994).

${ }^{9}$ E. Penev, S. Stojković, P. Kratzer, and M. Scheffler, Phys. Rev. B 69, 115335 (2004).

${ }^{10}$ P. Ballet, J. B. Smathers, H. Yang, C. L. Workman, and G. J. Salamo, J. Appl. Phys. 90, 481 (2001).

${ }^{11}$ C. Lobo and R. Leon, J. Appl. Phys. 83, 4168 (1998)

${ }^{12}$ D. Wasserman, S. A. Lyon, M. Hadjipanayi, A. Maciel, and J. F. Ryan, Appl. Phys. Lett. 83, 5050 (2003).

${ }^{13}$ E. Uccelli, S. Nürnberger, M. Bichler, G. Abstreiter, and A. Fontcuberta i Morral, Superlattices Microstruct. 44, 425 (2008). 